\title{
Shear-induced shift of spinodal line in entangled polymer blends
}

\author{
M. Criado-Sancho, ${ }^{1}$ D. Jou, ${ }^{2,3}$ J. Casas-Vázquez, ${ }^{2}$ and L. F. del Castillo ${ }^{4}$ \\ ${ }^{1}$ Departamento de Ciencias y Técnicas Físicoquímicas, Facultad de Ciencias, UNED, Senda del Rey, 9, 28040 Madrid, Spain \\ ${ }^{2}$ Departament de Física, Universitat Autònoma de Barcelona, 08193 Bellaterra, Catalonia, Spain \\ ${ }^{3}$ Institut d'Estudis Catalans, Carme 47, 08001 Barcelona, Catalonia, Spain \\ ${ }^{4}$ Departamento de Polímeros, Instituto de Investigaciones en Materiales, UNAM, Apartado Postal 70-360, México Distrito Federal, \\ 04510, Mexico
}

(Received 23 April 2002; published 30 December 2002)

\begin{abstract}
We study the shear-flow effects on phase separation of entangled polymer blends by incorporating into the chemical potential a nonequilibrium contribution due to the flow. The results are compared with those of a previous analysis by other authors which did not modify the chemical potential but used a different assumption for the stress tensor of the blend.
\end{abstract}

DOI: 10.1103/PhysRevE.66.061803

PACS number(s): 83.80.Tc, 64.75.+g, 05.70.Ln

Shear-induced effects in polymer solutions and blends are very interesting in thermodynamics, statistical mechanics, hydrodynamics, and engineering and, accordingly, much research is being carried out on them [1-3]. Clarke and McLeish [4] recently studied the shear-flow effects on phase separation of entangled polymer blends. They considered a two-fluid model, taking into account the effects of mutual friction among the chains, of shear stress, and of the inhomogeneities in the chemical potential of the components. They assumed that the chemical potential keeps its localequilibrium form (i.e., that it does not depend on the flow), and the shear-flow effects were entirely attributed to the coupling between the divergence of the viscous pressure and the diffusion flux. On this basis, they showed the possibility of a rich variety of changes of the phase diagrams of flowing polymer blends.

Our aim here is to present an alternative study by keeping for the stress tensor the usual upper-convected Maxwell form and incorporating nonequilibrium contributions into the chemical potentials. Such an approach has been used in the description of shear-induced diffusion and of phase separation in polymer solutions $[1,2,5-7]$. A comparison of the two approaches may be useful for discussion of the role of dynamical and thermodynamical effects in this active field of research.

First of all, we summarize the results by Clarke and McLeish [4], who use a two-fluid description proposed by Doi and Onuki [8]. The main result of this approach is the following expression for the evolution of the volume fraction $\phi_{A}$ of one of the polymers, say, polymer $A$ :

$$
\frac{\partial \phi_{A}}{\partial t}=-\boldsymbol{\nabla} \cdot\left(\mathbf{v} \phi_{A}\right)+\boldsymbol{\nabla} \cdot \boldsymbol{M} \cdot\left[\boldsymbol{\nabla}\left(\mu_{A}-\mu_{B}\right)-\alpha \boldsymbol{\nabla} \cdot \boldsymbol{\sigma}\right],
$$

where $\mathbf{v}$ is the volume average velocity, $\boldsymbol{M}$ the mobility tensor, $\boldsymbol{\sigma}$ the viscous stress tensor, and $\alpha$ a parameter depending on the ratio of entanglements in both polymers. Then the viscous stress is coupled to the diffusion flux $\mathbf{J}$, which is given by

$$
\mathbf{J}=-\boldsymbol{M} \cdot\left[\boldsymbol{\nabla}\left(\mu_{A}-\mu_{B}\right)-\alpha \boldsymbol{\nabla} \cdot \boldsymbol{\sigma}\right]
$$

In [4], this coupling plays an essential role in the shift of the spinodal line, whereas $\mu_{A}$ and $\mu_{B}$ are the localequilibrium chemical potentials of polymers $A$ and $B$, respectively. Thus, the shear-induced effects are attributed to a purely dynamical origin.

As the memory function for the stress relaxation of the blend, Clarke and McLeish take

$$
\begin{aligned}
G\left(t-t^{\prime}\right)= & \left(\phi_{A}\left\{G_{A} \exp \left[-\left(t-t^{\prime}\right) / \tau_{A}\right]\right\}^{1 / 2}\right. \\
& \left.+\phi_{B}\left\{G_{B} \exp \left[-\left(t-t^{\prime}\right) / \tau_{B}\right]\right\}^{1 / 2}\right)^{2},
\end{aligned}
$$

where $\phi_{i}, G_{i}$, and $\tau_{i}$ are, respectively, the volume fraction, plateau modulus, and relaxation time of polymer $i$. This nonlinear mixing rule, following from the model of double reptation $[9,10]$, is the simplest one to describe the details of coupled stress relaxation in polymer blends. It yields for the steady viscous stresses the following expressions:

$$
\begin{aligned}
\sigma_{x y}= & \dot{\gamma}\left[\phi_{A}^{2} G_{A} \tau_{A}+4 \phi_{A} \phi_{B}\left(G_{A} G_{B}\right)^{1 / 2} \frac{\tau_{A} \tau_{B}}{\tau_{A}+\tau_{B}}+\phi_{B}^{2} G_{B} \tau_{B}\right] \\
\equiv & G_{A} \tau_{A} \dot{\gamma} Y\left(\phi_{A}\right) \\
N_{1}= & \sigma_{x x}-\sigma_{y y}=2 \dot{\gamma}^{2}\left[\phi_{A}^{2} G_{A} \tau_{A}^{2}+8 \phi_{A} \phi_{B}\left(G_{A} G_{B}\right)^{1 / 2}\right. \\
& \left.\times\left(\frac{\tau_{A} \tau_{B}}{\tau_{A}+\tau_{B}}\right)^{2}+\phi_{B}^{2} G_{B} \tau_{B}^{2}\right] \\
\equiv & 2 G_{A}\left(\tau_{A} \dot{\gamma}\right)^{2} X\left(\phi_{A}\right)
\end{aligned}
$$

where $\dot{\gamma}$ is the shear rate, and $X\left(\phi_{A}\right)$ and $Y\left(\phi_{A}\right)$ are polynomials defined in order to have more compact expressions and whose explicit forms are given in Eqs. (A2) and (A3) in the Appendix.

The diagonal components of the stress tensor are assumed to have the form [4]

$$
\sigma_{x x}=\frac{2}{3} N_{1}, \quad \sigma_{y y}=\sigma_{z z}=-\frac{1}{3} N_{1},
$$

so that $\operatorname{Tr} \boldsymbol{\sigma}=0$. Note that the form (6) for the diagonal components of the viscous stresses is not unique, as one might 
assume. For instance, if an upper-convected Maxwell model [11] is used to describe the viscous stress tensor, it follows that

$$
\sigma_{x x}=N_{1}, \quad \sigma_{y y}=\sigma_{z z}=0 .
$$

Although the stress tensor can only be determined within an isotropic constant, the options (6) and (7) do not differ by a constant, but a variable, namely, $-\frac{1}{3} N_{1}$, and thus they are not equivalent.

The different choices (6) and (7) lead to very different results concerning the flow effects on the diffusion flux. Indeed, consider for instance the $y$ component of $\mathbf{J}$, which according to Eq. (2) is given by

$$
J_{y}=-M\left[\frac{\partial}{\partial y}\left(\mu_{A}-\mu_{B}\right)-\alpha\left(\frac{\partial \sigma_{x y}}{\partial x}+\frac{\partial \sigma_{y y}}{\partial y}+\frac{\partial \sigma_{z y}}{\partial z}\right)\right] .
$$

In the steady state, one has $\partial \sigma_{x y} / \partial x=0$ and $\partial \sigma_{z y} / \partial z=0$, and one is left with

$$
J_{y}=-M\left[\frac{\partial}{\partial y}\left(\mu_{A}-\mu_{B}\right)-\alpha \frac{\partial \sigma_{y y}}{\partial y}\right] .
$$

If Eq. (6) is assumed, $\sigma_{y y}=-\frac{1}{3} N_{1}$ and the coupling term contributes to $J_{y}$, whereas if Eq. (7) is considered, the coupling term does not contribute to the diffusion flux. This is the main difference between the approach in [4] and the present one. In [4], $\mu_{A}$ and $\mu_{B}$ do not depend on the flow and all the shear-induced effects are attributed to the coupling term. In the approach proposed in $[1,7,12,13]$ for dilute polymer solutions and applied here to polymer blends, $\mu_{A}$ and $\mu_{B}$ depend on the flow and the coupling term vanishes (for the plane Couette flow studied in this paper, but not in some other flows such as the cone-and-plate one $[14,15])$. Thus, from now on we will use Eq. (7) and a nonequilibrium contribution for $\mu$.

Anyway, we will follow the standard procedure $[4,8,12]$ of defining an effective diffusion coefficient $D_{\text {eff }}$ by rewriting Eq. (1) for the space Fourier transform of the perturbations $\delta \phi_{A}$ in the volume fraction of polymer $A$ in the form

$$
\frac{\partial \delta \phi_{A}}{\partial t}=-D_{\mathrm{eff}} q^{2} \delta \phi_{A}
$$

where $\mathbf{q}$ is the wave vector. For wave vectors in the $y$ and $z$ directions (when the velocity is in the $x$ direction and the velocity gradient in the $y$ direction), the effective diffusion coefficient is

$$
D_{\text {eff }}\left(q_{i}\right)=2 M\left[\chi_{c}-\chi+\kappa q_{i}^{2}+\Delta \chi_{c}\left(q_{i}\right)\right] \quad(i=y, z),
$$

where $\chi$ is the Flory-Huggins interaction parameter, which is a function of the temperature of the form

$$
\chi=\frac{1}{2}+\Psi\left(\frac{\Theta}{T}-1\right)
$$

where $\Psi$ and $\Theta$ are parameters that depend on the system, $\chi_{c}$ its value on the quiescent spinodal, and $\kappa$ the interfacial energy. The spinodal line indicating the onset of instability of the homogeneous phase is given by the condition $D_{\text {eff }}=0$. Indeed, for positive values of $D_{\text {eff }}$, inhomogeneities will tend to disappear, whereas negative values of $D_{\text {eff }}$ will enhance inhomogeneities $[8,12,13]$. Thus, $\Delta \chi_{c}$ describes the $\dot{\gamma}$-dependent shift in the spinodal line. When $\Delta \chi_{c}<0$, shearinduced demixing occurs, i.e., phase separation is enhanced, whereas $\Delta \chi_{c}>0$ corresponds to shear-induced mixing, which contributes to the stability of the one-phase system. In the approach by Clarke and McLeish [4], $\Delta \chi_{c}$ is given by

$$
\begin{gathered}
\Delta \chi_{c}\left(q_{y}\right)=\frac{2 \alpha}{3 k_{B} T}\left(\tau_{A} \dot{\gamma}\right)^{2} G_{A}\left[\frac{1}{2} X^{\prime}\left(\phi_{A}\right)-\frac{X\left(\phi_{A}\right) Y^{\prime}\left(\phi_{A}\right)}{Y\left(\phi_{A}\right)}\right], \\
\Delta \chi_{c}\left(q_{z}\right)=\frac{\alpha}{3 k_{b} T} G_{A}\left(\tau_{A} \dot{\gamma}\right)^{2} X^{\prime}\left(\phi_{A}\right),
\end{gathered}
$$

where $X^{\prime}$ and $Y^{\prime}$ denote the derivatives with respect to $\phi_{A}$. The results (13) have been taken as the basis for the analysis of some experiments by assuming an effective diffusion coefficient of the form $D_{\text {eff }}=D_{\text {eq }}-a \dot{\gamma}^{2}$, where $D_{\text {eq }}$ is the diffusion coefficient at equilibrium and $a$ is taken as a parameter to be identified by fitting the experimental data [16].

In contrast with [4], we include in the chemical potential contributions from the flow, which may be expressed in terms of the viscous stress in a macroscopic approach, or of the macromolecular configuration tensor in a more microscopic approach $[1,2,5-7,17]$. In particular, in extended irreversible thermodynamics the nonequilibrium contribution of the viscous stress tensor to the Gibbs free energy $\mathcal{G}$ is given by $[1,2,18-22,15]$

$$
\Delta \mathcal{G}=V \widetilde{J} \sigma_{x y}^{2}=v_{0}\left(n_{A} N_{A}+n_{B} N_{B}\right) \widetilde{J} \sigma_{x y}^{2},
$$

$V$ being the total volume, $\widetilde{J}$ the steady-state compliance (i.e., the ratio of the viscoelastic relaxation time to the shear viscosity), $n_{i}$ the number of moles of species $i$ per unit volume, $N_{i}$ the number of monomers of polymer $i(i=A, B)$, and $v_{0}$ the molar volume of the monomer, which in this simple illustration is supposed the same for $A$ and $B$, although the result could be straightforwardly generalized to different molar volumes. This contribution is supported by several microscopic arguments [1] and, in summary, it takes into account the contribution to the Gibbs free energy of the orientation and stretching of the macromolecules due to the flow. From Eq. (14), one may derive the nonequilibrium contributions to the chemical potentials,

$$
\begin{array}{r}
\Delta \mu_{i}=\left(\frac{\partial \Delta \mathcal{G}}{\partial n_{i}}\right)_{T, p, \sigma_{x y}}=v_{0} N_{j}\left[\widetilde{J}+\left(\delta_{i A}-\phi_{A}\right) \frac{\partial \widetilde{J}}{\partial \phi_{A}}\right] \sigma_{x y}^{2} \\
(i=A, B, \quad i \neq j),
\end{array}
$$

$T$ being the temperature, $p$ the pressure, and $\delta_{i j}$ the Kronecker delta. 
The explicit form of the steady-state compliance $\widetilde{J}$ for polymer blends may be obtained from the general formula [11]

$$
\widetilde{J}=\frac{\sum_{i} \tau_{i} \eta_{i}}{\left(\sum_{i} \eta_{i}\right)^{2}}=\frac{\sum_{i} \tau_{i}^{2} G_{i}}{\left(\sum_{i} G_{i} \tau_{i}\right)^{2}},
$$

and from the fact that the memory function $G(t)$ in Eq. (3) may be explicitly rewritten as

$$
\begin{aligned}
G(t)= & \sum_{i} G_{i}(t)=\phi_{A}^{2} G_{A} \exp \left(-t / \tau_{A}\right)+\phi_{B}^{2} G_{B} \exp \left(-t / \tau_{B}\right) \\
& +2 \phi_{A} \phi_{B}\left(G_{A} G_{B}\right)^{1 / 2} \exp \left(-t / \tau_{C}\right)
\end{aligned}
$$

where $\tau_{C}^{-1}=2\left(\tau_{A}^{-1}+\tau_{B}^{-1}\right)$. By combining Eqs. (16) and (17), it follows that

$$
\begin{aligned}
\widetilde{J} & =\frac{\phi_{A}^{2} \tau_{A}^{2} G_{A}+\phi_{B}^{2} \tau_{B}^{2} G_{B}+2 \phi_{A} \phi_{B} \tau_{C}^{2}\left(G_{A} G_{B}\right)^{1 / 2}}{\left[\phi_{A}^{2} \tau_{A} G_{A}+\phi_{B}^{2} \tau_{B} G_{B}+2 \phi_{A} \phi_{B} \tau_{C}\left(G_{A} G_{B}\right)^{1 / 2}\right]^{2}} \\
& =\frac{1}{G_{A}} \frac{X\left(\phi_{A}\right)}{Y\left(\phi_{A}\right)^{2}} .
\end{aligned}
$$

Therefore, the following expressions for the nonequilibrium contribution (15) to the chemical potentials are obtained:

$$
\begin{gathered}
\Delta \mu_{A}=v_{0} N_{A} G_{A}\left(\tau_{A} \dot{\gamma}\right)^{2}\left[\frac{Z\left(\phi_{A}\right)+W\left(\phi_{A}\right)}{Y\left(\phi_{A}\right)}\right] \\
\Delta \mu_{B}=v_{0} N_{B} G_{A}\left(\tau_{A} \dot{\gamma}\right)^{2} \frac{Z\left(\phi_{A}\right)}{Y\left(\phi_{A}\right)}
\end{gathered}
$$

where $Z\left(\phi_{A}\right)$ and $W\left(\phi_{A}\right)$ are polynomials given in the Appendix [Eqs. (A4) and (A10), respectively].

Therefore, the total shift of the spinodal line becomes in our model

$$
\begin{aligned}
\Delta \chi_{c}\left(q_{y}\right)= & \frac{v_{0} N_{A}}{2 k_{B} T} G_{A}\left(\tau_{A} \dot{\gamma}\right)^{2}\left[\left(-3 W Y^{\prime}+Y W^{\prime}\right)\right. \\
& \left.+(1-\lambda)\left(-3 Z Y^{\prime}+Y Z^{\prime}\right)\right] Y^{-2},
\end{aligned}
$$

when we consider the $y$ direction (where $\sigma_{x y}=$ const) and

$$
\begin{aligned}
\Delta \chi_{c}\left(q_{z}\right)= & \frac{v_{0} N_{A}}{2 k_{B} T} G_{A}\left(\tau_{A} \dot{\gamma}\right)^{2}\left[\left(-W Y^{\prime}+Y W^{\prime}\right)\right. \\
& \left.+(1-\lambda)\left(-Z Y^{\prime}+Y Z^{\prime}\right)\right] Y^{-2}
\end{aligned}
$$

in the $z$ direction (along which $\dot{\gamma}=$ const). The parameter $\lambda$ is defined as the ratio of the number of monomers, $N_{A}$ and $N_{B}$, in the polymeric chains $A$ and $B$, i.e., $\lambda=N_{B} / N_{A}$.

Note that, as well as Eqs. (13a) and (13b), the results (21) and (22) point out that in the presence of the flow one should consider an effective diffusion coefficient of the form $D_{\text {eff }}$ $=D_{\text {eq }}-a^{\prime} \dot{\gamma}^{2}$, with $a^{\prime}$ a coefficient different from the one obtained in the Clarke and McLeish theory, but yielding similar qualitative results.

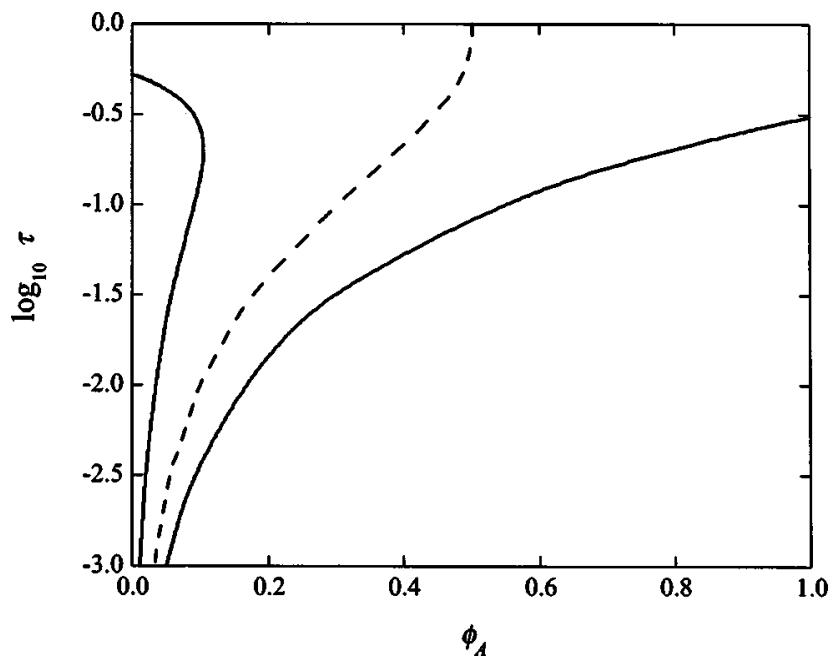

FIG. 1. Borders of instability in the $y$ direction when $G=1$. The continuous curve is calculated from Eq. (21) when $\lambda=1$ and the dashed curve corresponds to the results [4] calculated from Eq. (13a).

In Fig. 1 we compare our results (21) with (13a) obtained in [4]. In this figure is plotted the border of the instability behavior, namely, $\Delta \chi_{c}=0$, in a diagram of $\log _{10} \tau$ vs $\phi$, for $G=1$. In the Clarke and McLeish results, such a border is a single line, whereas in our model two different lines arise. The zones where $\Delta \chi_{c}<0$ correspond to shear-induced demixing, whereas where $\Delta \chi_{c}>0$ the shear induces mixing and it enhances stability. The physical differences between the predictions of Clarke and Mc Leish and ours are directly seen: in the former, the nonequilibrium and equilibrium spinodal lines cross over each at a single point, whereas in the latter there are crossings at two points, depending on the range of $\tau$ and $\phi_{A}$ considered. It is also noted that, although $\lambda$ appears in Eq. (21), calculations reveal only a minor influence on $\Delta \chi_{c}\left(q_{y}\right)$ in our results (21), whereas it does not even appear in Eq. (13a). The limit when $\tau \ll 1$ would correspond to an entangled polymer solution of chains $A$ in solvent $B$; in this case, the shear flow induces mixing and enhances stability. When $\tau$ increases, i.e., when the viscoelastic effects of the solvent $B$ increase, a demixing behavior appears. Following Clarke and McLeish [4], the parameter $\alpha$ can be written as

$$
\alpha=\frac{1-\lambda^{*}}{\left(1-\lambda^{*}\right) \phi_{A}+\lambda^{*}}
$$

with

$$
\lambda *=\lambda \frac{N_{A, \text { ent }}}{N_{B, \text { ent }}}
$$

where $N_{i, \text { ent }}$ is the degree of polymerization of an entanglement segment in polymer $i$. The last equation shows that $\alpha$ takes values near zero when $A$ and $B$ have equal degrees of polymerization and its entanglement behavior is similar $(\tilde{\lambda}$ $=1)$. In this situation, the $\Delta \chi_{c}$ proposed in [4] vanishes as follows from Eqs. (13a) and (13b). 


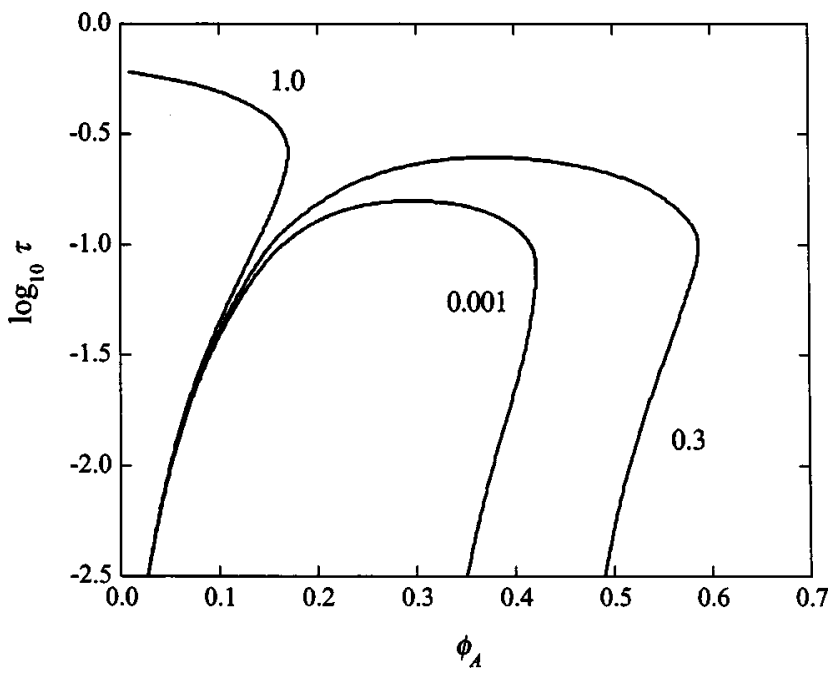

FIG. 2. Borders of instability in the $z$ direction when $G=1$. All the curves have been calculated by means of Eq. (22) using different values of the parameter $\lambda$, which are indicated on the respective curves. For the considered value of $G$, Eq. (13b) always predicts $\Delta \chi_{c}<0$, as was pointed out in Ref. [4].

In Fig. 2 we compare the results for $\Delta \chi_{c}\left(q_{z}\right)$ in our approach, namely, Eq. (22), and in that of Clarke and McLeish Eq. (13b) for $G=1$. In contrast with the method for obtaining $\Delta \chi_{c}\left(q_{y}\right)$, where the gradient of $\mu_{A}-\mu_{B}$ is carried out at constant $\sigma_{x y}$, in the analysis of $\Delta \chi_{c}\left(q_{z}\right)$ this gradient is performed at constant shear rate $\dot{\gamma}$. Thus, the "spinodal line" is no longer univocal, but depends on the direction of observation. One difference of $\Delta \chi_{c}\left(q_{z}\right)$ with respect to $\Delta_{\chi_{c}}\left(q_{y}\right)$ is that the former is sensitive to the ratio $\lambda\left(\equiv N_{B} / N_{A}\right)$ whereas the second one remains practically unchanged under modifications of $\lambda$.

When a $T-\phi_{A}$ diagram is considered (Figs. 3 and 4), in the zones where $\Delta \chi_{c}<0$ the spinodal line is shifted toward higher temperatures [according to the relation (12) between $\chi$ and $T$ ], whereas where $\Delta \chi_{c}>0$ it is shifted in the opposite direction. In order to apply the previous results to a real system, we consider one of the isotopic blends of poly(dimethylsiloxane) (PDMS) studied by Beaucage et al. [23]. In this reference, neutron scattering experiments are reported which yield the values of the Flory-Huggins parameter and its dependence on temperature. The system that we have chosen is the blend in which the degree of polymerization of hydrogenous PDMS is 964 and that of deuterated PDMS is 957 (whose density is of the order of $9.7 \times 10^{-3} \mathrm{~kg} \mathrm{~m}^{-3}$ ) for which $\Psi=0.5013$ and $\Theta=1.17 \mathrm{~K}$. Taking into account the values of the quoted degrees of polymerization, one has $\lambda$ $\approx 1$ and $N_{A}=957$. From the rheological quantities reported by Migler [24] we have estimated $G_{A}=1.5 \times 10^{4} \mathrm{~Pa}$ and $\tau_{A}$ $=2.0 \times 10^{-3} \mathrm{~s}$. Note that in Fig. 3, both the Clarke and McLeish (CM) model and the extended irreversible thermodynamics (EIT) model predict an increase of the critical temperature of the same order. However, the shift of the value of the critical concentration is opposite in the two models: it is positive in the CM model and negative in the EIT model. In contrast, in the situation reported in Fig. 4, EIT predicts a shear-induced increase in critical temperature whereas the

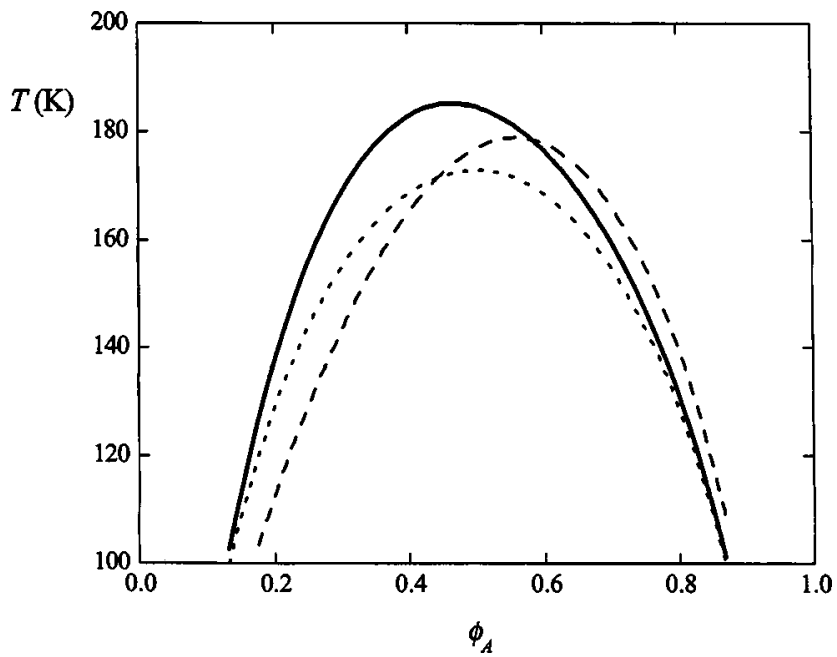

FIG. 3. Spinodal lines predicted for the isotopic blend described in the text. The dotted curve corresponds to the Flory-Huggins model without interfacial contributions. The other curves are associated with fluctuations in the $y$ direction when the system is submitted to a shear stress $\sigma_{x y}=250 \mathrm{~Pa}$. The continuous curve and the dashed curve are calculated from Eqs. (21) and (13a), respectively. The calculations have been carried out using $G=1, \log _{10} \tau$ $=-0.5$, and $\lambda^{*}=0.5$.

CM model predicts a decrease; note, furthermore, that the shift predicted by the CM model is more sensitive to the value of the shear rate than is the shift predicted by EIT. Experimental analysis of these spinodal lines could allow one to establish which of these two theoretical models is more suitable.

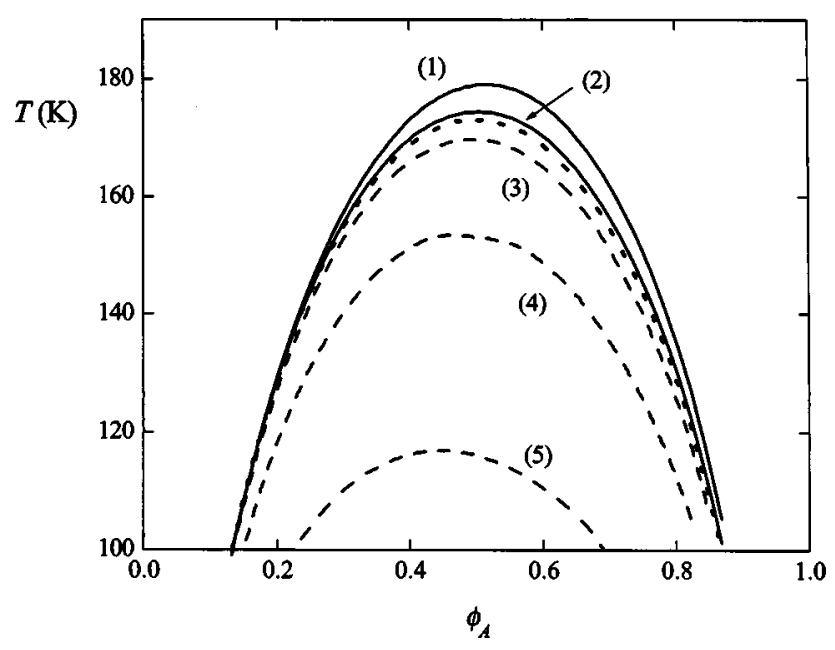

FIG. 4. Spinodal lines predicted for the isotopic blend described in the text. The dotted curve corresponds to the Flory-Huggins model without interfacial contributions. The other curves are associated with fluctuations in the $z$ direction taking $G=1, \log _{10} \tau$ $=-0.5$, and $\lambda=1$ when the system is submitted to a constant shear rate. The continuous curves are calculated from Eq. (22) considering two shear rates $\dot{\gamma}=10$ (curve 1) and $5 \mathrm{~s}^{-1}$ (curve 2). All dashed curves correspond to Eq. (13b): $\lambda *=0.9$ and $\dot{\gamma}=5 \mathrm{~s}^{-1}$ (curve 3), $\lambda *=0.5$ and $\dot{\gamma}=5 \mathrm{~s}^{-1}$ (curve 4), and $\lambda^{*}=0.5$ and $\dot{\gamma}=10 \mathrm{~s}^{-1}$ (curve 5). 
In summary, we have pointed out here two different descriptions of the shear-induced shift of the spinodal line, due to the coupling between $\mathbf{J}$ and $\boldsymbol{\nabla} \cdot \boldsymbol{\sigma}$ and the nonequilibrium contribution to the chemical potentials $\mu$. Whereas in the plane Couette flow studied here the coupling term disappears when the upper-convected Maxwell model is used, this is not so in other flow geometries, such as for instance the coneand-plate model. In such situations, the two contributions should be added, as was done, for instance, in [13]. Indeed, in addition to the relative importance of the latter contributions to the steady-state nonequilibrium spinodal line outlined here, other good motivations to consider them arise in the analysis of time-dependent phenomena, such as, for instance, the rate of shear-induced separation. Indeed, in [14] it was shown (in a polymer dilute solution in a cone-and-plate experiment) that by keeping only the coupling between viscous shear and diffusion flux (i.e., by ignoring the nonequilibrium contributions to the chemical potential) one predicts a shear-induced polymer separation which is two to three orders of magnitude slower than the one experimentally observed. Instead, inclusion of nonequilibrium contributions such as Eq. (14) yields the correct order of magnitude for this separation time, since the separation is accelerated because of a thermodynamical instability due to the nonequilibrium terms of the chemical potential [13]. The analysis of similar experiments in polymer blends would be of much interest in clarifying the physical relevance of the several kinds of nonequilibrium contribution to the effective diffusion coefficient.

We acknowledge financial support from the Spanish Ministry of Science and Technology under Grant No. BFM20000351-C03-01 and from the Direcció General de Recerca of the Generalitat of Catalonia under Grant No. 2001 SGR 00186. Also, one of us (L.F.C.) acknowledges the support of the Dirección General de Asuntos de Personal Académico of the UNAM (México) under Grant No. IN-119200.

\section{APPENDIX}

In order to obtain the explicit form of the polynomials quoted in the previous sections, the following parameters are introduced:

$$
G=\frac{G_{B}}{G_{A}}, \quad \tau=\frac{\tau_{B}}{\tau_{A}}, \quad \Lambda=\frac{\tau}{1+\tau},
$$

which allow us to write for $X\left(\phi_{A}\right)$ and $Y\left(\phi_{A}\right)$ appearing in Eqs. (4) and (5),

$$
\begin{gathered}
X\left(\phi_{A}\right)=\tau^{2} G+\left(8 \Lambda^{2} G^{1 / 2}-2 \tau^{2} G\right) \phi_{A} \\
+\left(1-8 \Lambda^{2} G^{1 / 2}+\tau^{2} G\right) \phi_{A}^{2}, \\
Y\left(\phi_{A}\right)=\tau G+\left(4 \Lambda G^{1 / 2}-2 \tau G\right) \phi_{A}+\left(1-4 \Lambda G^{1 / 2}+\tau G\right) \phi_{A}^{2} .
\end{gathered}
$$
be

The polynomial $Z\left(\phi_{A}\right)$ in Eqs. (19) and (20) is found to

$$
Z\left(\phi_{A}\right)=z_{0}+z_{1} \phi_{A}+z_{2} \phi_{A}^{2}+z_{3} \phi_{A}^{3}+z_{4} \phi_{A}^{4},
$$

with coefficients given by

$$
\begin{gathered}
z_{0}=\tau^{3} G^{2} \\
z_{1}=12 \tau^{2} \Lambda G^{3 / 2}-6 \tau^{3} G^{2} \\
z_{2}=\left(-\tau+64 \Lambda^{3}+5 \tau^{2}\right) G+\left(-24 \tau \Lambda^{2}-36 \tau^{2} \Lambda\right) G^{3 / 2} \\
+12 \tau^{3} G^{2}, \\
z_{3}=\left(4 \Lambda+32 \Lambda^{2}\right) G^{1 / 2}+\left(-2 \tau-8 \tau^{2}-160 \Lambda^{3}\right) G \\
+\left(48 \tau \Lambda^{2}+36 \tau^{2} \Lambda\right) G^{3 / 2}-10 \tau^{3} G^{2} \\
z_{4}=3+\left(-12 \Lambda-24 \Lambda^{2}\right) G^{1 / 2}+\left(3 \tau+3 \tau^{2}+96 \Lambda^{3}\right) G \\
+\left(-24 \tau \Lambda^{2}-12 \tau^{2} \Lambda\right) G^{3 / 2}+3 \tau^{3} G^{2}
\end{gathered}
$$
(19),

Analogously, we can write for $W\left(\phi_{A}\right)$ appearing in Eq.

$$
W\left(\phi_{A}\right)=w_{0}+w_{1} \phi_{A}+w_{2} \phi_{A}^{2}+w_{3} \phi_{A}^{3},
$$

with the following coefficients:

$$
\begin{gathered}
w_{0}=\left(8 \tau \Lambda^{2}-8 \tau^{2} \Lambda\right) G^{3 / 2}+2 \tau^{3} G^{2} \\
w_{1}=\left(2 \tau-4 \tau^{2}-32 \Lambda^{3}\right) G+24 \tau^{2} \Lambda G^{3 / 2}-6 \tau^{3} G^{2} \\
w_{2}=-24 \Lambda^{2} G^{1 / 2}+\left(6 \tau^{2}+96 \Lambda^{3}\right) G \\
+\left(-24 \tau \Lambda^{2}-24 \tau^{2} \Lambda\right) G^{3 / 2}+6 \tau^{3} G^{2}, \\
w_{3}=-2+\left(8 \Lambda+16 \Lambda^{2}\right) G^{1 / 2}+\left(-2 \tau-2 \tau^{2}-64 \Lambda^{3}\right) G \\
+\left(16 \tau \Lambda^{2}+8 \tau^{2} \Lambda\right) G^{3 / 2}-2 \tau^{3} G^{2}
\end{gathered}
$$

[1] D. Jou, J. Casas-Vázquez, and M. Criado-Sancho, Thermodynamics of Fluids under Flow (Springer, Berlin, 2000).

[2] D. Jou, J. Casas-Vázquez, and M. Criado-Sancho, Adv. Polym. Sci. 120, 207 (1995).

[3] A. Onuki, J. Phys.: Condens. Matter 9, 6119 (1997).

[4] N. Clarke and T. C. B. McLeish, Phys. Rev. E 57, R3731 (1998).
[5] C. Rangel-Nafaile, A. B. Metzner, and K. F. Wissbrun, Macromolecules 17, 1187 (1984).

[6] B. Wolf, Macromolecules 17, 615 (1984).

[7] M. Criado-Sancho, D. Jou, and J. Casas-Vázquez, Polymer 36, 4107 (1995); Phys. Rev. E 56, 1887 (1997).

[8] M. Doi and A. Onuki, J. Phys. II 2, 1631 (1992).

[9] J. des Cloizeaux, Europhys. Lett. 5, 437 (1988). 
[10] J. D. Ferry, Viscoelastic Properties of Polymers (Wiley, New York, 1980).

[11] R. B. Bird, C. F. Curtiss, R. C. Armstrong, and O. Hassager, Dynamics of Polymeric Liquids (Wiley, New York, 1987), Vol. 2.

[12] J. Casas-Vázquez, D. Jou, and M. Criado-Sancho, Europhys. Lett. 23, 469 (1993).

[13] L. F. del Castillo, M. Criado-Sancho, and D. Jou, Polymer 41, 2633 (2000); M. Criado-Sancho, D. Jou, L. F. del Castillo, and J. Casas-Vázquez, ibid. 41, 8425 (2000).

[14] M. J. Mc Donald and S. J. Muller, J. Rheol. 40, 259 (1996).

[15] J. Casas-Vázquez, L. F. del Castillo, D. Jou, and M. CriadoSancho, Phys. Rev. E 63, 057101 (2001).

[16] H. Gerard, J. S. Higgins, and N. Clarke, Macromolecules 37, 5411 (1999).

[17] A. Onuki, Phys. Rev. Lett. 62, 2472 (1989); J. Phys. Soc. Jpn.
59, 3423 (1990); 59, 3427 (1990).

[18] D. Jou, J. Casas-Vázquez, and G. Lebon, Extended Irreversible Thermodynamics, 3rd ed. (Springer, Berlin, 2001); Rep. Prog. Phys. 51, 1105 (1988); 62, 1035 (1999).

[19] R. E. Nettleton and S. L. Sobolev, J. Non-Equilib. Thermodyn. 20, 205 (1995); 21, 297 (1996).

[20] L. S. García-Colín and F. J. Uribe, J. Non-Equilib. Thermodyn. 16, 89 (1991).

[21] I. Müller and T. Ruggeri, Extended Thermodynamics (Springer, New York, 1993).

[22] Extended Thermodynamic Systems, edited by S. Sieniutycz and P. Salamon (Taylor and Francis, New York, 1992).

[23] G. Beaucage, S. Sukurmaran, S. J. Clarson, M. S. Kent, and D. W. Schaefer, Macromolecules 29, 8349 (1996).

[24] K. B. Migler, J. Rheol. 44, 277 (2000). 